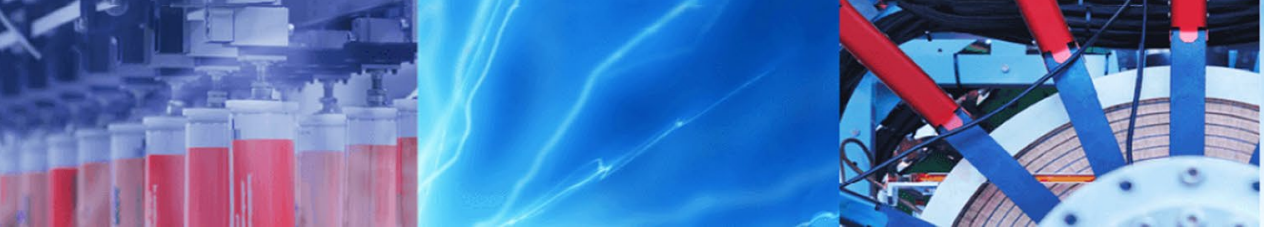

Research Article

\title{
In-silico approach to understand the inhibition of corrosion by some potent triazole derivatives of pyrimidine for steel
}

\author{
Bello Abdullahi Umar ${ }^{1}$. Adamu Uzairu
}

(c) Springer Nature Switzerland AG 2019

\begin{abstract}
Density functional theory (DFT) with B3LYP functional and 6-31G* basis set was performed on ten triazole derivatives of pyrimidine used as corrosion inhibitors for steel in $1.0 \mathrm{M} \mathrm{HCl}$ solution which led to the optimization of their structures, generation of electronic and other important Quantum chemical descriptors using spartan'14 Software. The computations were carried out in non-protonated and protonated forms. The obtained results show a good correlation between the chemical structures of the inhibitors and their experimental inhibition efficiencies (\%IEs). The ranking of these efficiencies (\%IEs) nicely matched with the order of a good number of the generated descriptors but with a varying degree of correlation as the majority of the descriptors indicates that I-4 is the best inhibitor among the data set. Molecular dynamic simulations were further carried out on the studied inhibitors with Fe (110) surface using Material Studio 8.0. The obtained results of MD simulations suggest that the interaction was as a result of the chemical adsorption on the steel surface since the binding energy is $>100 \mathrm{kcal} \mathrm{mol}^{-1}$ for all the inhibitors and the best adsorption energy was found to be $-333.783 \mathrm{kcal} \mathrm{mol}^{-1}(\mathrm{I}-4)$. This observation is in good agreement with the DFT results and the experimental findings.
\end{abstract}

Keywords Triazole derivatives of pyrimidine $\cdot$ DFT (B3LYP/6-31G*) $\cdot$ MD simulation

\section{Introduction}

Steel is the most commonly used material in many industries, like oil, gas and chemical industries, it came to be used for most purposes where wrought iron was formerly used $[1,2]$. Nonetheless, the major problem associated with its use seems to reach its high susceptibility to corrosion in acidic solutions, which results in a considerable loss in its essential properties. To adopt effective preventive methods against corrosion of metals, various strategies have been applied to control this undesirable phenomenon such as materials selection, cathodic protection, coatings and the use of corrosion inhibitors. The inhibitors have been considered as the most practical and easiest method [3]. Typically, these organic compounds along with heteroatoms and lone pair have shown good inhibition performance for many metals and rapid progress in recent years has been made in the design and synthesis of a variety of compounds such as quinoles, quinoxalines, pyridinium, triazoles, and imidazolines, etc. [4]. They seem to act by adsorbing onto the surface of the metal, blocking one or more of the undesirable reactions occurring at the solution/metal interface and therefore causing high protection of metal against dissolution $[5,6]$.

Experimental techniques like weight-loss method, electrochemical impedance spectroscopy, potentiodynamic polarization, etc. $[7,8]$, have been used to understand the corrosion process and its inhibition. Though experiments mostly are time-consuming, costly, and lacking in explaining the mechanism of inhibition of the corrosion $[9,10]$. In recent years, to understand some unknown properties during the interaction of inhibitors with the metal surface. Density Functional Theory based calculations have been proved to be a very powerful tool for studying the electronic and structural properties of inhibitors. The geometry of the inhibitor molecule in its ground state and the

Bello Abdullahi Umar, abdallahbum@yahoo.com | 'Department of Chemistry, Ahmadu Bello University, Zaria, Nigeria.

SN Applied Sciences (2019) 1:1413 | https://doi.org/10.1007/s42452-019-1451-y 
nature of their molecular orbitals (HOMO; Highest Occupied Molecular Orbital and LUMO; Lowest Unoccupied Molecular Orbital) are directly involved in the inhibitive properties of these molecules. Such calculations, though instructive, are not always adequate for providing more clarification of the experimental results. Modeling of an experiment by molecular dynamic (MD) simulations have been accepted as an effective and authentic technique. MD simulations are particularly well-poised to investigate the structure of the corrosion inhibitors and the mechanism of inhibition at an atomic level under a variety of conditions [11].

Gonzalez-Olvera et al. [12] have recently investigated corrosion inhibition effect of some triazole derivatives of pyrimidine on the steel surface in $1.0 \mathrm{M} \mathrm{HCl}$ solution employing electrochemical impedance spectroscopy method. The obtained experimental results have reflected that these inhibitors are remarkably good corrosion-resistant inhibitors in $1.0 \mathrm{M} \mathrm{HCl}$ medium. In getting a complete picture of the mechanism of inhibition action of these compounds as well as to get an explanation of their inhibition efficiency trend, quantum chemical calculation and MD simulation are carried out in this current study. Also, correlations among the observed molecular parameters and experimentally obtained inhibition efficiency outcomes have been performed.

\section{Computational details}

\subsection{Selection of inhibitors}

A set of ten Triazole Derivatives as corrosion inhibitors were selected from the literature [12] and used for this present study. The 2D structure of each inhibitor in the data set was drawn using ChemDraw ultra V12.0. The structures of the inhibitors and their experimental inhibition efficiency (\%IE) are presented in Table 1.

\subsection{Molecular optimization and quantum chemical calculation}

The 2D structure of each inhibitor was converted to the 3D structure using Spartan 14.0 software. The molecular mechanic force field $(\mathrm{MM}+)$ was used to clean and minimize the structures using of SPARTAN'14 software on Dell Intel(R)Core(TM)i7-5500U CPU), 16.00 GB RAM @ $2.400 \mathrm{GHz}$ $2.400 \mathrm{GHz}$ processor on Windows 8.1 Pro 64-bit Operating system, $\times 64$-based processor, so as to remove all the strain on the structures of the inhibitor molecules. Additionally, this guarantees a fully-defined and stable conformation of the studied inhibitor molecules of this study $[13,14]$. Geometry optimization was set at ground state utilizing the DFT at the B3LYP level of theory and 6-311G (d) basis set. The generated chemical parameters were Energy of highest occupied molecular orbital (E-HOMO), energy of lowest unoccupied molecular orbital (E-LUMO), dipole moment $(\mu)$ and some others were calculated such as energy bandgap $(\Delta E)$, chemical hardness $(\eta)$, chemical softness $(\sigma)$, global electronegativity $(\chi)$, number of transferred electrons $(\Delta N)$ using relevant relations (Eqs. 1-5) as reported before in literature $[15,16]$.

$\Delta E=E_{\text {LUMO }}-E_{\text {HOMO }}$

$\eta=-\frac{1}{2}\left(E_{\text {HOMO }}-E_{L U M O}\right)$

$\sigma=\frac{1}{\eta}=-\left(\frac{2}{E_{\text {HOMO }}-E_{L U M O}}\right)$

$\chi=\frac{-(E-H O M O+E-L U M O)}{2}$

$\Delta N=\frac{\chi_{F e}-\chi_{i n h}}{2\left(\eta_{F e}+\eta_{i n h}\right)}$

where $\chi_{\mathrm{Fe}}=7 e \mathrm{e}, \eta_{\mathrm{Fe}}=0[17]$.

\subsection{Molecular dynamics (MD) simulation}

The simulation (MD) was applied to portray the adsorption mechanism between the studied inhibitors and the steel surface using Materials studio version 8.0 material simulation program from Biovia. The inhibitors were optimized and modeled employing COMPASS force field. The system (simulation) was carried out at $298 \mathrm{~K}$ using Andersen thermostat with NVE microcanonical ensemble at a time step of $1.0 \mathrm{fs}$ and the simulation time was set as $5 \mathrm{ps}$. The simulation was conducted in a box (simulation-box) of $(24.82 \AA \times 24.82 \AA \times 45.27 \AA)$ under periodic boundaries conditions. The simulation box contains a Fe (110) slab, solvent (acid) molecules $\left(\mathrm{H}_{2} \mathrm{O}+\mathrm{H}_{3} \mathrm{O}^{+}+\mathrm{Cl}^{-}\right)$and an inhibitor. Fe (110) surface was chosen as the studied Fe surface because $\mathrm{Fe}(110)$ was the most density packed Fe surface and stable $[18,19]$. The Fe crystal has ten (10) layers out of which seven (7) down layers were frozen. The adsorption and binding energies values were calculated using Eqs. 6 and 7 respectively [16].

$E_{\text {adsorption }}=E_{\text {Total }}-\left(E_{\text {Surface }+\mathrm{H}_{2} \mathrm{O}+\mathrm{H}_{3} \mathrm{O}^{+}+\mathrm{Cl}^{-}}+E_{\text {Inhibitor }}\right)$

$E_{\text {binding }}=-E_{\text {adsorption }}$

where $E_{\text {Total }}$ is $E_{\text {Surface }+\mathrm{H}_{2} \mathrm{O}+\mathrm{H}_{3} \mathrm{O}^{+}+\mathrm{Cl}^{-}+\text {lnhibitor }}$ (the total energy of the corrosion system) $E_{\text {Surface }}+\mathrm{H}_{2} \mathrm{O}+\mathrm{H}_{3} \mathrm{O}^{+}+\mathrm{Cl}^{-}$is the energy 
Table 1 Triazole derivatives of pyrimidine nucleobases and their inhibition efficiency (\%IE)<smiles></smiles><smiles>O=c1ccn(Cc2cn(Cc3ccc(I)cc3)nn2)c(=O)[nH]1</smiles><smiles>Cc1cn(Cc2cn(Cc3ccccc3)nn2)c(=O)[nH]c1=O</smiles><smiles>Cc1cn(Cc2cn(Cc3ccc(F)cc3)nn2)c(=O)[nH]c1=O</smiles><smiles>Cc1cn(Cc2cn(Cc3ccc(Cl)cc3)nn2)c(=O)[nH]c1=O</smiles> 
of the Fe surface together with $\mathrm{H}_{2} \mathrm{O}+\mathrm{H}_{3} \mathrm{O}^{+}+\mathrm{Cl}^{-}$molecules and $E_{\text {Inhibitor }}$ is the energy of the inhibitor.

\section{Results and discussion}

\subsection{Quantum chemical studies of the neutral form of the inhibitors}

To examine the inhibitive performance of the inhibitors concerning the molecular structures, some DFT (quantum) parameters were generated to explain their mode of adsorption. The geometry optimized molecular structure, the distributions of HOMO and LUMO of the first inhibitor are depicted in Fig. 1. Table 4 present the results of DFT parameters of the studied inhibitors like the E-HOMO, E-LUMO, dipole moment $(\mu)$, energy band gap $(\Delta E)$, chemical hardness ( $\eta)$, chemical softness ( $\sigma)$, global electronegativity $(\chi)$ and number of transferred electrons $(\Delta N)$ computed using Spartan 14 software at B3LYP level of theory, 6-31G $(d, p)$ basis set and some relevant equations.

The HOMOs of the studied inhibitors are mainly dominated on the pi-bond and then delocalized over the whole aromatic Pyrimidine ring in each molecule as seen in Fig. 1. The Halogen substituents in each of the inhibitors from $\mathrm{I}-2$ to $\mathrm{I}-10$ are also involved in the HOMO. Pyrimidine ring of inhibitor 1 in Fig. 1 shows a significant contribution of the two nitrogen atoms, pi-bond and the two oxygen atoms of the -dione to the distributions of HOMO and LUMO. The HOMO electron density distribution of the studied inhibitors indicate favorable interactions of the inhibitors with an electron lacking metallic orbitals. The distribution of the LUMO electron densities of all the studied inhibitors was also spread all over on the Pyrimidine rings. The electronic surfaces of $\mathrm{HOMO}$ and LUMO revealed the Pyrimidine ring has the potential to donate and accept electrons under favorable conditions. This typical behavior is suitable for donor-acceptor interactions which may be responsible for the adsorption of inhibitors on the steel surface.

Based on the frontier orbital (molecular) approximation, the donor/acceptor relation occurs in between HOMOs and LUMOs (frontiers (molecular) orbitals) of reacting molecules $[20,21]$. The adsorption mechanism of an inhibitor on a metallic surface usually increases with the increase of the HOMO energy and decreases with the decrease of LUMO energy. Therefore, the inhibitor molecule can donate their lone pair of electrons from the HOMO orbital to the $d$-orbital of the metallic molecule and also the inhibitor molecule will receive the electrons coming from the metallic $d$-orbital through the LUMO orbital for in-electron-donation and electron-back-donation method. Therefore, E-HOMO has always related with electron donation of an inhibitor; a high value of E-HOMO shows the high tendency of an inhibitor molecule to donate lone pair of electrons to the d-orbital of the acceptor metal.

From Table 2, the values of E-HOMO for the studied inhibitors increases in the order $\mathrm{I}-4>\mathrm{I}-3>\mathrm{I}-5>\mathrm{I}-2>\mathrm{I}-1$ $>\mathrm{I}-9>\mathrm{I}-10>\mathrm{I}-8>\mathrm{I}-7>\mathrm{I}-6$ respectively. The trend in the increase of the HOMO energies show some level of consistency with the increase of the efficiencies (inhibition), Thus, I-4 with a high efficiency (96.1\%) has a larger HOMO energy and I- 6 with the lowest efficiency (90.2\%) has a lower HOMO energy as seen in Table 2. Conversely, a low value of E-LUMO of an inhibitor molecule demonstrates the high tendency of the inhibitor molecule to receive electrons coming from the metallic $d$-orbital during the
Fig. 1 a Optimized structure, b HOMO distribution and c LUMO distribution for $\mathrm{I}-1$ (Inh.1)
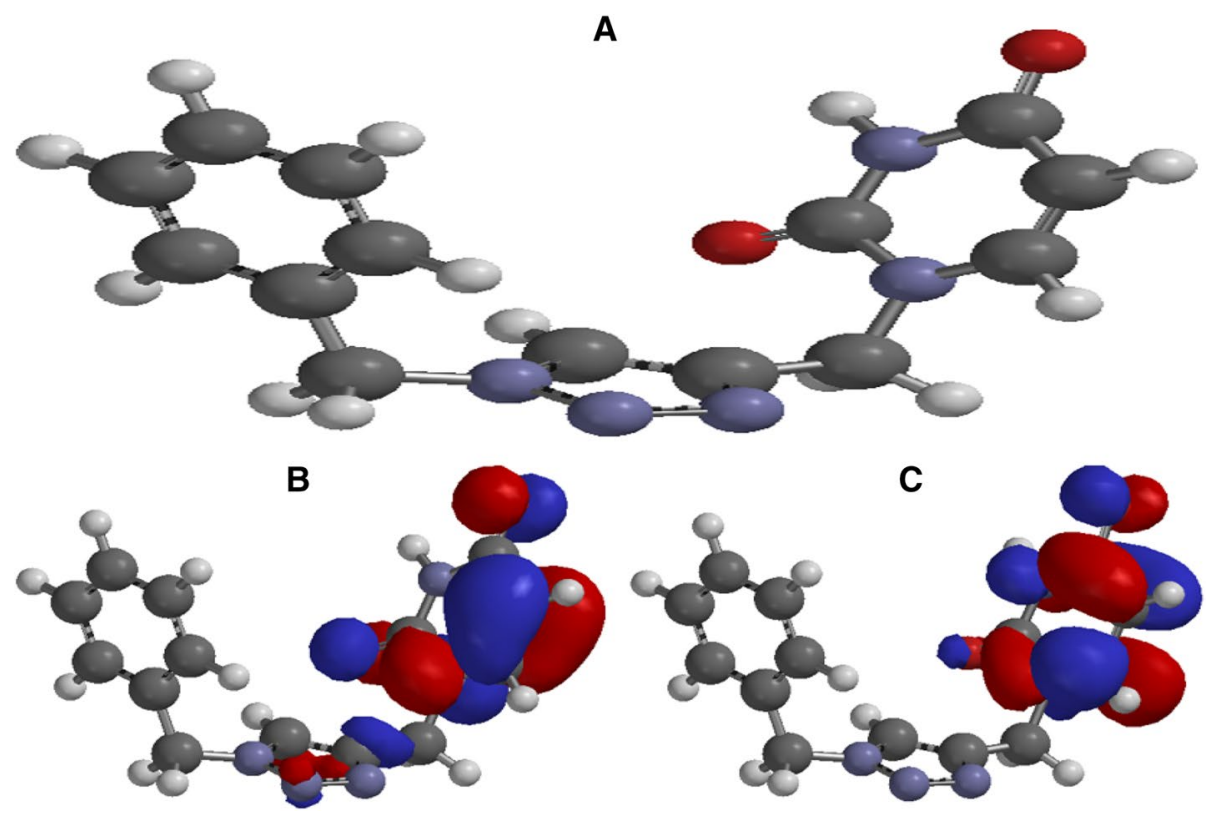

SN Applied Sciences 
Table 2 Quantum chemical parameters of the neutral form of the studied inhibitors

\begin{tabular}{lllllllll}
\hline $\mathrm{S} / \mathrm{N}$ & $\mathrm{E}-\mathrm{HOMO}(\mathrm{eV})$ & $\mathrm{E}-\mathrm{LUMO}(\mathrm{eV})$ & $\Delta \mathrm{E}$ & $\mu$ (Debye) & $\eta$ & $\sigma$ & $\chi$ & $\Delta \mathrm{N}$ \\
\hline 1 & -6.59 & -1.04 & 5.55 & 3.38 & 2.775 & -0.360 & 3.815 & 4.419 \\
2 & -6.63 & -1.07 & 5.56 & 3.85 & 2.780 & -0.359 & 3.850 & 4.379 \\
3 & -6.66 & -1.10 & 5.56 & 4.04 & 2.780 & -0.359 & 3.880 & 4.337 \\
4 & -6.66 & -1.09 & 5.57 & 4.03 & 2.785 & -0.359 & 3.875 & 4.352 \\
5 & -6.65 & -1.08 & 5.57 & 3.79 & 2.785 & -0.359 & 3.865 & 4.365 \\
6 & -6.32 & -0.90 & 5.42 & 3.99 & 2.710 & -0.369 & 3.610 & 4.593 \\
7 & -6.38 & -0.95 & 5.43 & 3.13 & 2.715 & -0.368 & 3.665 & 4.527 \\
8 & -6.39 & -0.96 & 5.43 & 2.01 & 2.715 & -0.368 & 3.675 & 4.513 \\
9 & -6.41 & -0.98 & 5.43 & 1.92 & 2.715 & -0.368 & 3.695 & 4.487 \\
10 & -6.40 & -1.09 & 5.31 & 2.06 & 2.655 & -0.377 & 3.745 & 4.321 \\
\hline
\end{tabular}

The energy of highest occupied molecular orbital (E-HOMO), Energy of lowest unoccupied molecular orbital (E-LUMO) Energy bandgap $(\Delta \mathrm{E})$, dipole moment $(\mu)$ chemical hardness $(\eta)$, chemical softness $(\sigma)$, global electronegativity $(\chi)$, number of transferred electrons $(\Delta N)$ back-donation process. From Table 2, the E-LUMO value in the ascending order are: $\mid-6>1-7>1-8>1-9>1-1>1-2>1-5>$ $\mathrm{I}-4>\mathrm{I}-10>\mathrm{I}-3$. This indicates that $\mathrm{I}-3$ has a stronger chance of accepting electrons from the orbital of the metal as they interact with the Fe surface. Therefore, I-3 may strongly adsorb on the surface of the metallic and achieve better inhibition effectiveness though the second highest in terms of \%IE. I-6 strongly agrees with EIS findings as it has the lowest \%IE having the highest LUMO energy.

Energy band gap, $\Delta \mathrm{E}$ is another crucial property as most efficient inhibitors were characterized by a small value of $\Delta E$ [22]. In Table 2, the trend at which the energy gap decreases is not consistent with the increase in the experimental \%IEs of the studied inhibitors. However, the reverse is the case i.e. the $\Delta \mathrm{E}$ increases when the \% IEs decreases. The dipole moment $(\mu)$ is another parameter which is commonly used for the prediction of the direction of the inhibition process. It shows a distribution of the electrons on the molecule, and also measure the polarity in any given bond [23]. The molecules with higher $\mu$ values tend to form a strong interaction (dipole-dipole) with the metallic steel surface, resulting to the strong adsorption of the inhibitor on the metallic surface and hence resulting in the better IE\% of an inhibitor [4]. Nevertheless, there is no established correlation between the $\mu$ and IE\% reported [19]. The results shown in Table 2 indicate that I-3 has the largest $\mu$ value and I-9 has the lowest $\mu$ value. Among all the ten (10) studied inhibitors, increasing the $\mu$ leads to an increment of their IE\% due to the increase in the intermolecular forces.

Inhibitor with the large hardness $(n)$ value is expected to be weaker compared to other inhibitors with lower values [24]. Thus, as shown in Table 2, the hardness value increases as the \%IE increases. I-4 has the highest $\eta$ value hence the best inhibitor and this agrees with the experimental observation reported in Table 1. The larger $\sigma$ value indicates that the inhibitor has a softer nature and will have higher chances of donating electrons to the metallic molecule [25]. The arrangement across the inhibitors in the $\sigma$ values as presented in Table 2 decreases in the order, $\mathrm{I}-2>\mathrm{I}-3>\mathrm{I}-4>\mathrm{I}-5>\mathrm{I}-1>\mathrm{I}-7>\mathrm{I}-7$ $>1-8>1-9>1-6>1-10$. This suggests that $\mathrm{I}-2$ has the highest and therefore the most reactive inhibitor molecule.

The electronegativity $(\chi)$ describes the electron attracting the power of an inhibitor molecule. The High value of $\chi$ shows the strong attracting capability of an inhibitor molecule to accept the electrons from the metallic (Fe) surface [9]. Afterward, the inhibitors that have larger electronegativities would have better interaction with Fe surface and then will have better IE\%. From Table 2 , it can be observed that the $\chi$ values of the ten inhibitors obey the order $\mathrm{I}-3>\mathrm{I}-4>\mathrm{I}-5>\mathrm{I}-2>\mathrm{I}-1$ $>\mathrm{I}-10>\mathrm{I}-9>\mathrm{I}-8>\mathrm{I}-7>\mathrm{I}-6$, hence, it is confirmed that $\mathrm{I}-3$ followed by $1-4$ has the higher tendency of accepting electrons and I-6 has the least among the studied inhibitors and these findings are in good agreement with the experimental findings. The values of $\Delta N$ demonstrate the fraction of electrons that transfer from the inhibitor to the metallic surface. If $\Delta \mathrm{N}>0$, then electrons were transferred from the inhibitor molecule to the Fe-surface while if $\Delta \mathrm{N}<0$, then electrons were transferred from Fesurface to inhibitor molecule through electron back donation process [16]. From Table 2, it can be observed that the values of $\Delta N$ of the studied inhibitors are all greater than $0(\Delta N>0)$, this indicates that all the studied inhibitors may donate electrons on to the Fe surface through the formation of a coordinate covalent bond. Examination of the results in Table 2 indicates that the values of $\Delta N$ of the inhibitors have a very little difference. This further confirms the electron-donating ability of the studied inhibitors as observed from their experimental findings. 


\subsection{Quantum chemical studies of the neutral form of the inhibitors}

Presence of heteroatoms in the structures of the inhibitor molecules suggests a high tendency towards of protonation in the acidic medium. Thus, the reactivity of the inhibitor molecules in their protonated forms should be considered. Geometry optimization of the protonated form of the inhibitors are carried out and it is observed that from Table 3, E-HOMO values for both the inhibitors are shifted towards the more negative value in comparison to the E-HOMO values of neutral form. It signifies that in the protonated form, electron donation capability of the inhibitor molecules is decreased and it is also expected as after protonation inhibitor molecules are not capable enough to donate their electrons to the metallic surfaces. This fact is further confirmed by the fraction of electron transferred from the inhibitors to the metallic surface $(\Delta N)$. Inspection of Table 3 shows that all the values of $\Delta N$ for Fe (110) surface are lower than in the neutral form of the inhibitors.

On the other hand, if we look at the E-LUMO values it can be seen that E-LUMO values are also shifted towards the more negative values than its neutral form, it pointing out electron acceptance capability in protonated form is increased. It is further counter supported by the electronegativity values of the inhibitors. Comparison of electronegativity values of the two forms of inhibitors molecules reflects that electronegativity values in the protonated form of inhibitor molecules are much higher than that of the neutral form. It reflects electron attraction capability from the metal surface is increased by the protonated form of inhibitor molecules. Therefore, after a complete analysis of both the form of inhibitors molecules it can be concluded that the neutral form of inhibitors molecule has higher tendency to donates their electrons and protonated form of inhibitors molecules have superior tendency to accept electrons. Now, it is clear from the above mention discussion that a more complete analysis of reactivity is obtained from both the form of inhibitor molecules.

\subsection{Molecular dynamic simulation studies}

Electronic parameters alone are not enough to determine the trend of the inhibitive performances of these studied inhibitors despite its success in explaining the mechanism of action of the inhibitors. Thus, it is essential to conduct thorough modeling of the direct interactions of the studied inhibitors with the Fe surface. It is a belief that the major mechanism of the inhibition of corrosion is through adsorption. Therefore, adsorption of these inhibitors on the steel $(\mathrm{Fe})$ surface was simulated to predict the nature of interactions between the studied inhibitors and Fe (110) crystal surface in $1.0 \mathrm{M} \mathrm{HCl}$. The first step of this simulation process was the geometry optimization of the studied inhibitors, solvent (acid) molecules $\left(\mathrm{H}_{2} \mathrm{O}, \mathrm{H}_{3} \mathrm{O}^{+}\right.$, and $\left.\mathrm{Cl}^{-}\right)$ and $\mathrm{Fe}(110)$ crystal. The geometry optimization of the corrosion system was conducted until the total energy of the system reached a local minimum on the potential energy surface. During the optimization process, the atomic coordinates were adjusted using COMPASS force-field which continues unless the total energies of the individual structures reached to the minimum energy, after which, a box was created for the simulation process by importing the Fe (110) crystal, solvent molecules $\left(\mathrm{H}_{2} \mathrm{O}, \mathrm{H}_{3} \mathrm{O}^{+}\right.$and $\left.\mathrm{Cl}^{-}\right)$ and the inhibitor molecule. Then the MD process was conducted and the system attained equilibrium when both the energy and temperature of the system were balanced. All the other inhibitors were studied similarly. The equilibrium configurations of the simulated system for the first inhibitor are depicted in Fig. 2. It could be observed that
Table 3 Quantum chemical parameters of the protonated form of the studied inhibitors

\begin{tabular}{lllllllll}
\hline $\mathrm{S} / \mathrm{N}$ & E-HOMO $(\mathrm{eV})$ & $\mathrm{E}-\mathrm{LUMO}(\mathrm{eV})$ & $\Delta \mathrm{E}$ & $\mu$ (Debye) & $\eta$ & $\sigma$ & $\chi$ & $\Delta \mathrm{N}$ \\
\hline 1 & -6.9621 & -1.9343 & 5.0278 & 4.5253 & 2.5139 & 0.3977883 & 4.4482 & 3.207485 \\
2 & -7.0021 & -1.9643 & 5.0378 & 4.9953 & 2.5189 & 0.39699869 & 4.4832 & 3.1697838 \\
3 & -7.0321 & -1.9943 & 5.0378 & 5.1853 & 2.5189 & 0.39699869 & 4.5132 & 3.1320003 \\
4 & -7.0321 & -1.9843 & 5.0478 & 5.1753 & 2.5239 & 0.39621221 & 4.5082 & 3.144527 \\
5 & -7.0221 & -1.9743 & 5.0478 & 4.9353 & 2.5239 & 0.39621221 & 4.4982 & 3.1571465 \\
6 & -6.6921 & -1.7943 & 4.8978 & 5.1353 & 2.4489 & 0.4083466 & 4.2432 & 3.3755638 \\
7 & -6.7521 & -1.8443 & 4.9078 & 4.2753 & 2.4539 & 0.40751457 & 4.2982 & 3.3149735 \\
8 & -6.7621 & -1.8543 & 4.9078 & 3.1553 & 2.4539 & 0.40751457 & 4.3082 & 3.302704 \\
9 & -6.7821 & -1.8743 & 4.9078 & 3.0653 & 2.4539 & 0.40751457 & 4.3282 & 3.278165 \\
10 & -6.7721 & -1.9843 & 4.7878 & 3.2053 & 2.3939 & 0.41772839 & 4.3782 & 3.1381635 \\
\hline
\end{tabular}

The energy of highest occupied molecular orbital (E-HOMO), Energy of lowest unoccupied molecular orbital (E-LUMO) Energy bandgap $(\Delta E)$, dipole moment $(\mu)$ chemical hardness $(\eta)$, chemical softness $(\sigma)$, global electronegativity $(\chi)$, number of transferred electrons $(\Delta N)$ 
Fig. 2 Equilibrium interaction configurations of the first inhibitor on Fe (110) surface

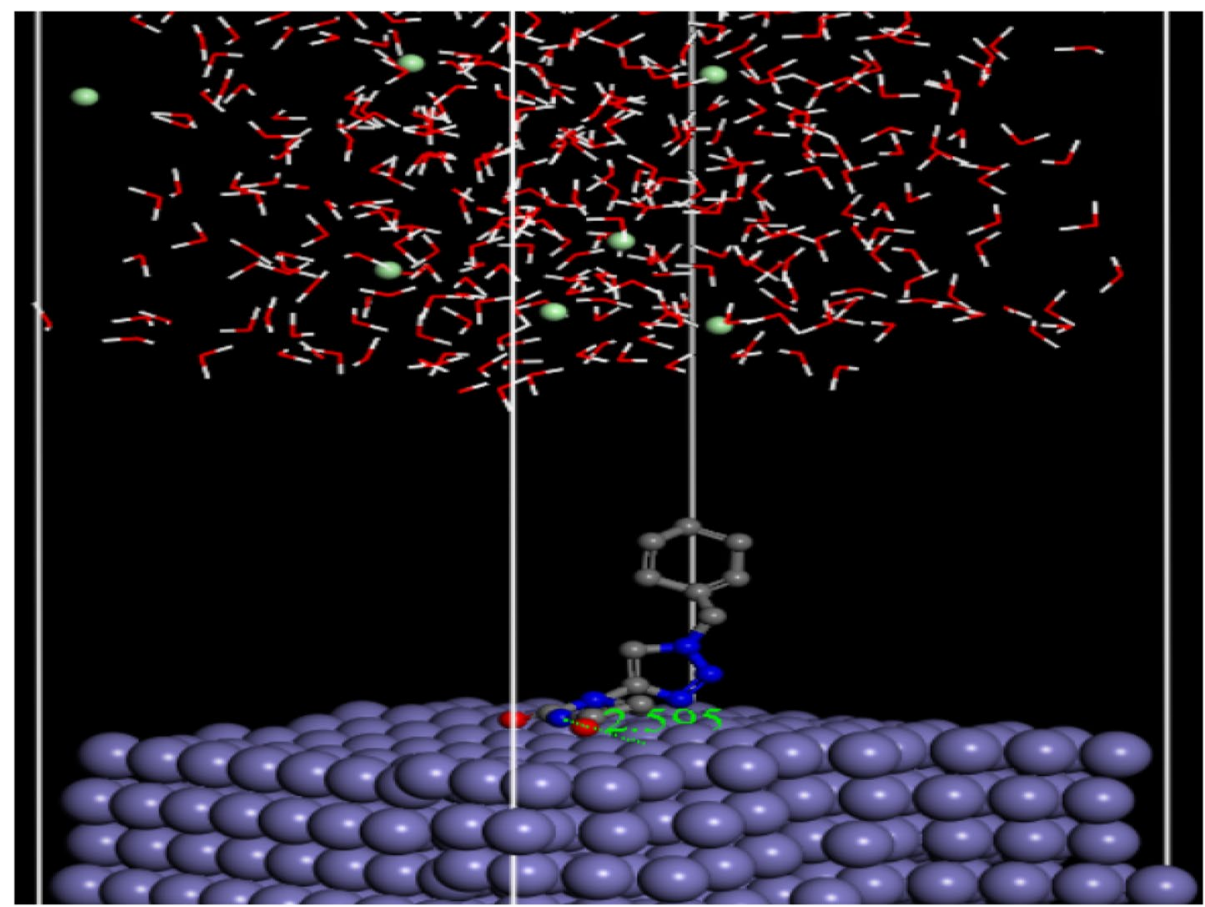

in Fig. $3 a$, $b$ in the middle of the MD simulation process the system moves towards the equilibrium. After the system attained an equilibrium, the adsorption and the binding energy values of the inhibitors adsorbed on the Fe surface was calculated according to Eqs. (6) and (7) respectively and are presented in Table 4.

It can be observed from the molecular structures of the examined inhibitors that these molecules contain various electrons (lone pair) on the $\mathrm{N}$ and $\mathrm{O}$ atoms and also $\pi$-bonds of the aromatic systems. Therefore, these electrons (lone pair) on the heteroatoms will be donated to an empty d orbital of Fe Metal. It could be observed in Fig. 2, that the inhibitor adsorbs almost flatly in orientation with the Fe surface and the interaction is more through the pyrimidine ring. This flat orientation of the inhibitor molecule on the Fe surface can be as a result of a chemical bond formation between the inhibitor and Fe surface. This observation was supported by the HOMOs and LUMOs distributions of these inhibitors as the region (pyrimidine ring) was found to be the most active center of adsorption (see Fig. 1). Additionally, it could be seen from the results presented in Table 4 that the values of the adsorption energy of the simulation systems at $298 \mathrm{~K}$ were large and negative. These high negative values of adsorption energies may be attributed to the strong interaction which occurs between these inhibitors and Fe surfaces. Thus, the calculated adsorption energy values reveal that inhibitor 4 adsorbs on the Fe surface more spontaneously than any other inhibitor among the series. However, the adsorption capability of an inhibitor molecule on the Fe surface may also be determined from the values of the binding energy. The larger the binding energy is, the better will be the adsorption. Therefore, it is further confirmed from the results presented in Table 4, that all the adsorption of the inhibitors on the Fe surfaces occurred through chemical adsorption process since the magnitude of the binding energies is actually within the range of chemisorption $\left(>100 \mathrm{kcal} \mathrm{mol}^{-1}\right)$ [26].

Generally, a bond distance measured within $3.5 \AA$ describes a strong chemical bond formation between the atoms and the bond distance greater than $3.5 \AA$ signifies interaction between the atoms are of Van der Waals type $[27,28]$. Figure $3 b$ indicates the bond distance (shortest) between the $\mathrm{N}$-atom of the pyrimidine ring of the first inhibitor and Fe surface. The measured bond distances for all the studied inhibitors and Fe-surface were shown in Table 4. From the mentioned values in Table 4, it can be seen that all the measured bond distances are in the range (3.5 $\AA$ ), this indicates that chemical bond was formed between these inhibitors and the Fe surface. Thus, chemical adsorption occurred on Fe surfaces. These findings are consistent with the experimental results obtained from EIS measurement [12] as well as from quantum chemical studies. This could be explained from the molecular structures of the studied inhibitors and the distribution of the HOMOs and LUMOs. Inhibitors having halogen atoms in their molecular structures are more and easily adsorbed on metallic surfaces and shows better efficiency (inhibition). It was clear from the structures given in Table 1 that inhibitor $1-5$ has the similar pattern with the 


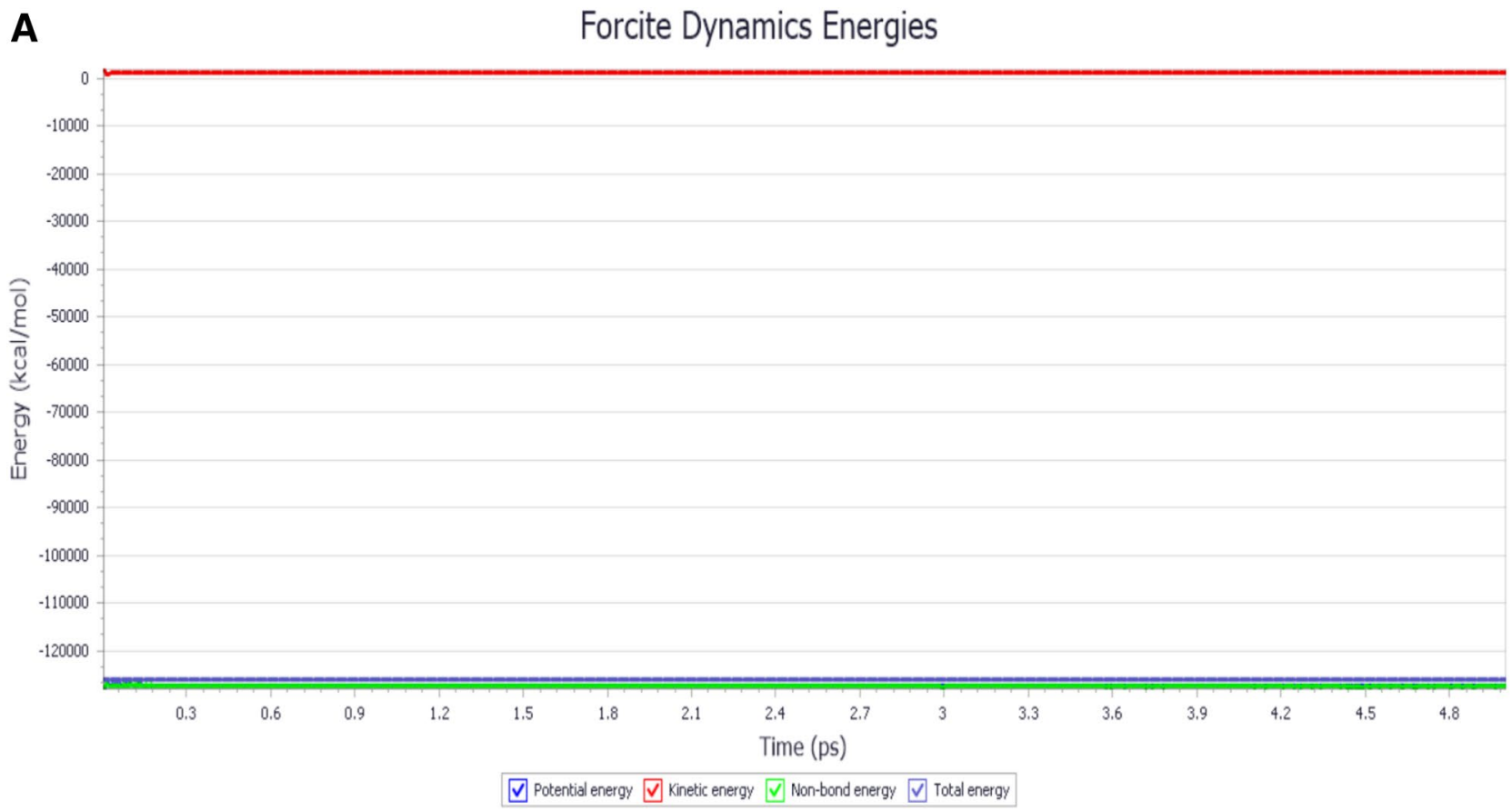

B

Forcite Dynamics Temperature

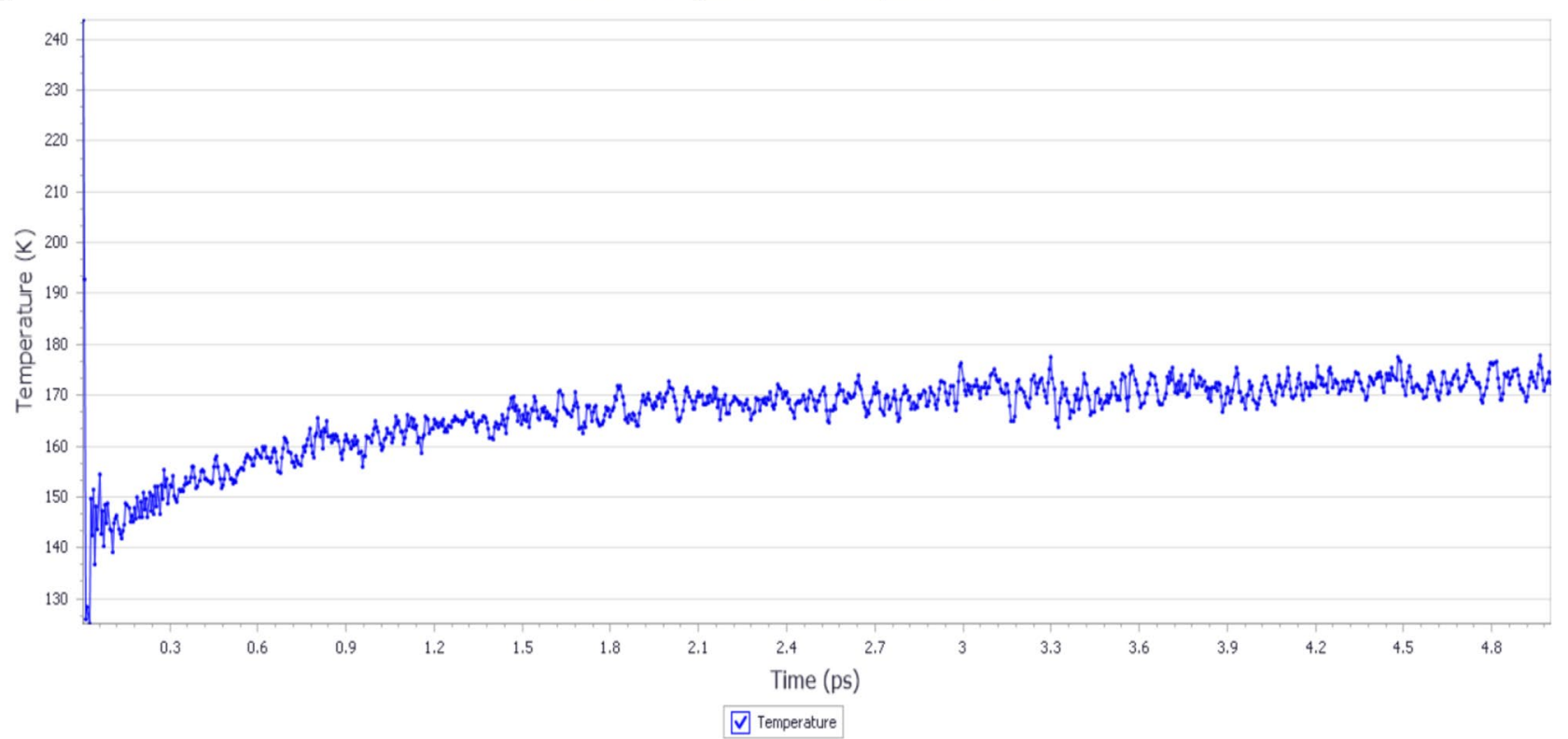

Fig. 3 a Fluctuation curves of energies obtained from MD simulation for the first inhibitor, $\mathbf{b}$ equilibrium temperature curve recorded from MD simulation for the first inhibitor

introduction of halogen atoms which increases the inhibition efficiency in the order $\mathrm{Br}>\mathrm{Cl}^{>} \mathrm{F}$ while the iodine atom slightly decreased the \%IE. For that reason, inhibitor-4 with bromine atom as the substituent was the best corrosion inhibitor among the studied inhibitors. Similarly, inhibitor 6-10 had a similar pattern with $\mathrm{F}, \mathrm{Cl}$ and $\mathrm{Br}$ and methyl group as substituent have the same effect in increasing the inhibition efficiency while the increase with iodine as the substituent is also slightly lower. In this regard the inhibitors with $\mathrm{Br}, \mathrm{F}$ or $\mathrm{Cl}$ are regarded to be the most potential corrosion inhibitors for steel.

Furthermore, to check the affection of the solvent (acidic) solution on the adsorption process of the system, the adsorption behavior of the first inhibitor in vacuum was simulated via MD simulation. The adsorption energy obtained in acidic solution is much less than

\section{SN Applied Sciences}


Table 4 Molecular dynamic simulations results of the studied inhibitors

\begin{tabular}{|c|c|c|c|c|c|c|}
\hline $\mathrm{S} / \mathrm{N}$ & $\mathrm{E}_{\mathrm{Fe}+\text { soln. }}\left(\mathrm{kcal} \mathrm{mol}{ }^{-1}\right)$ & $\mathrm{E}_{\text {Inh. }}\left(\mathrm{kcal} \mathrm{mol}^{-1}\right)$ & $\mathrm{E}_{\text {Total }}\left(\mathrm{kcal} \mathrm{mol}^{-1}\right)$ & $\mathrm{E}_{\text {Ads. }}\left(\mathrm{kcal} \mathrm{mol}^{-1}\right)$ & $\mathrm{E}_{\text {Bind. }}\left(\mathrm{kcal} \mathrm{mol}^{-1}\right)$ & $\mathrm{BD}(\AA ̊)$ \\
\hline 1 & $-125,832.513$ & -42.694 & $-126,081.177$ & -205.970 & 205.970 & 2.595 \\
\hline 2 & $-125,832.513$ & -40.231 & $-126,097.889$ & -225.145 & 225.145 & 2.891 \\
\hline 3 & $-125,832.513$ & -40.376 & $-126,111.548$ & -238.659 & 238.659 & 2.390 \\
\hline 4 & $-125,832.513$ & -40.323 & $-126,206.619$ & -333.783 & 333.783 & 2.160 \\
\hline 5 & $-125,832.513$ & -40.284 & $-126,074.778$ & -201.981 & 201.981 & 3.18 \\
\hline 6 & $-125,832.513$ & -78.975 & $-126,080.170$ & -168.682 & 168.682 & 2.920 \\
\hline 8 & $-125,832.513$ & -78.997 & $-126,144.664$ & -233.154 & 233.154 & 2.783 \\
\hline 9 & $-125,832.513$ & -75.505 & $-126,120.266$ & -212.248 & 212.248 & 2.842 \\
\hline 9 & $-125,832.513$ & -72.986 & $-126,082.620$ & -177.121 & 177.121 & 2.780 \\
\hline 10 & $-125,832.513$ & -74.047 & $-126,118.326$ & -211.766 & 211.766 & 3.050 \\
\hline
\end{tabular}

$\mathrm{E}_{\mathrm{Fe}+\text { soln. }}$ (energy of $\mathrm{Fe}+\mathrm{H}_{2} \mathrm{O}+\mathrm{H}_{3} \mathrm{O}^{+}+\mathrm{Cl}^{-}$), $\mathrm{E}_{\text {Inh. }}$ (energy of the inhibitor), $\mathrm{E}_{\mathrm{Total}}$ (total energy of the simulation system), $\mathrm{E}_{\mathrm{Ads}}$. (adsorption energy), $\mathrm{E}_{\mathrm{Bind}}$. (binding energy), $\mathrm{BD}$ (bond distance)

those obtained in the vacuum. This could be due to the less influential effect in vacuum and the complex nature of the corrosion process in solution, like adsorption of the solvent (acid) molecules, the affection of the acid solution, most especially the anions, that have been proved to have a high influence on adsorption of the inhibitor [29]. It was found that the adsorption energy of this inhibitor $\left(-1811.528 \mathrm{kcal} \mathrm{mol}^{-1}\right)$ changed significantly compared with the one simulated in acidic solution $\left(-419.28 \mathrm{kcal} \mathrm{mol}^{-1}\right)$. So also, the adsorption behavior is not consistent with those in the acid solution. Therefore, considering a solvent in the molecular dynamics simulation process is very necessary.

\section{Conclusion}

The Computational approach employed in this study was successful in explaining the inhibition performance of the studied inhibitors on the steel surface and has offered an atomic level understanding of the experimental findings. Quantum chemical calculation shows that the reactive sites in the structure of the inhibitors are majorly the $\mathrm{N}$-atom of the pyrimidine ring, $\pi$-electron centers, and halogen substituent. Electron-donation and electron-acceptance capability of these inhibitors were consistent with the results obtained from the experimental results. The interaction energies ( $E_{\text {ads. }}$ and $E_{\text {bind }}$ ) between the inhibitors and $\mathrm{Fe}(110)$ metal surface via MD-simulation were large indicating chemical bond formation and that the inhibitors can more tightly adsorb on the iron surface. Inhibitor 4 showed high adsorption energy $\left(-333.783 \mathrm{kcal} \mathrm{mol}^{-1}\right)$ among other co-inhibitors as observed from the experiment. The shortest bond distances between the heteroatoms of the inhibitors and Fe atoms in MD-simulation are lying within a range of $3.5 \AA$ which indicates that these inhibitors have a very strong interaction with steel $(\mathrm{Fe})$ surface. In conclusion, the DFT and MD simulation results are in good agreement with the experimental results. Thus, DFT along with MD simulation may be a very powerful tool for the designing of potent corrosion inhibitors and in the prediction of their inhibition performance before the synthesis.

Acknowledgements The authors sincerely acknowledge Ahmadu Bello University, Zaria and all the members of the Physical and Computational Chemistry research group for their advice and encouragement in the cause of this research.

\section{Compliance with ethical standards}

Conflict of interest The authors have declared they have no conflict of interest.

Ethical standard This article does not contain any studies with human or animal subjects.

\section{References}

1. Chaouiki A et al (2018) Understanding corrosion inhibition of mild steel in acid medium by new benzonitriles: insights from experimental and computational studies. J Mol Liq 266:603-616

2. Negm NA et al (2018) Amide type nonionic surfactants: synthesis and corrosion inhibition evaluation against carbon steel corrosion in acidic medium. J Mol Liq 256:574-580

3. Jiang $L$ et al (2018) Excellent corrosion inhibition performance of novel quinoline derivatives on mild steel in $\mathrm{HCl}$ media: experimental and computational investigations. J Mol Liq 255:53-63

4. Verma C et al (2016) Adsorption behavior of glucosamine-based, pyrimidine-fused heterocycles as green corrosion inhibitors for mild steel: experimental and theoretical studies. J Phys Chem C 120(21):11598-11611

5. Bedair M (2016) The effect of structure parameters on the corrosion inhibition effect of some heterocyclic nitrogen organic compounds. J Mol Liq 219:128-141 
6. Sayed GH et al (2018) Pyrazole, pyrazolone and enaminonitrile pyrazole derivatives: synthesis, characterization and potential in corrosion inhibition and antimicrobial applications. J Mol Liq 252:329-338

7. Zhang $D-Q$ et al (2008) Inhibition effect of some amino acids on copper corrosion in $\mathrm{HCl}$ solution. Mater Chem Phys 112(2):353-358

8. Amin MA, Ibrahim MM (2011) Corrosion and corrosion control of mild steel in concentrated $\mathrm{H} 2 \mathrm{SO} 4$ solutions by a newly synthesized glycine derivative. Corros Sci 53(3):873-885

9. Wazzan NA, Obot I, Kaya S (2016) Theoretical modeling and molecular level insights into the corrosion inhibition activity of 2-amino-1,3,4-thiadiazole and its 5-alkyl derivatives. J Mol Liq 221:579-602

10. Usman B, Jimoh I, Umar BA (2019) Theoretical study of 2-(3,4-dihydroxyphenyl) chroman-3,5,7-triol on corrosion inhibition of mild steel in acidic medium. Appl J Environ Eng Sci 5(1):66-74

11. Salarvand $Z$ et al (2017) Enhanced corrosion resistance of mild steel in $1 \mathrm{M} \mathrm{HCl}$ solution by trace amount of 2-phenyl-benzothiazole derivatives: experimental, quantum chemical calculations and molecular dynamics (MD) simulation studies. Corros Sci 114:133-145

12. González-Olvera R et al (2013) Multicomponent click synthesis of new 1,2,3-triazole derivatives of pyrimidine nucleobases: promising acidic corrosion inhibitors for steel. Molecules 18(12):15064-15079

13. Viswanadhan VN et al (1989) Atomic physicochemical parameters for three dimensional structure directed quantitative structure-activity relationships. 4. Additional parameters for hydrophobic and dispersive interactions and their application for an automated superposition of certain naturally occurring nucleoside antibiotics. J Chem Inf Comput Sci 29(3):163-172

14. Arthur DE et al (2016) QSAR modelling of some anticancer PGI50 activity on HL-60 cell lines. Albanian J Pharm Sci 3(1):4-9

15. Nwankwo HU et al (2016) Synthesis, characterization, antimicrobial studies and corrosion inhibition potential of 1,8-dimethyl-1,3,6,8,10,13-hexaazacyclotetradecane: experimental and quantum chemical studies. Materials 9(2):107

16. Saha SK, Banerjee P (2015) A theoretical approach to understand the inhibition mechanism of steel corrosion with two aminobenzonitrile inhibitors. RSC Adv 5(87):71120-71130

17. Musa AY, Jalgham RT, Mohamad AB (2012) Molecular dynamic and quantum chemical calculations for phthalazine derivatives as corrosion inhibitors of mild steel in $1 \mathrm{M} \mathrm{HCl}$. Corros Sci $56: 176-183$

18. Khaled K (2008) Molecular simulation, quantum chemical calculations and electrochemical studies for inhibition of mild steel by triazoles. Electrochim Acta 53(9):3484-3492
19. Nwankwo HU, Olasunkanmi LO, Ebenso EE (2017) Experimental, quantum chemical and molecular dynamic simulations studies on the corrosion inhibition of mild steel by some carbazole derivatives. Sci Rep 7(1):2436

20. Bereket G, Öğretir C, Özşahin Ç (2003) Quantum chemical studies on the inhibition efficiencies of some piperazine derivatives for the corrosion of steel in acidic medium. J Mol Struct (Thoechem) 663(1-3):39-46

21. Bello A, Uzairu A, Shallangwa G (2018) Molecular modelling and dynamic simulation of corrosion inhibitors on steel in acidic medium. FUW Trends Sci Technol J 3(2A):365-372

22. Ebenso E et al (2012) Quantum chemical investigations on quinoline derivatives as effective corrosion inhibitors for mild steel in acidic medium. Int J Electrochem Sci 7:5643-5676

23. Abdallah M et al (2013) Synergistic effect of some halide ions on the inhibition of zinc corrosion in hydrochloric acid by tetrahydro carbazole derivatives compounds. Int J Electrochem Sci 8:10001-10021

24. Murulana LC et al (2012) Experimental and quantum chemical studies of some bis (trifluoromethyl-sulfonyl) imide imidazolium-based ionic liquids as corrosion inhibitors for mild steel in hydrochloric acid solution. Ind Eng Chem Res 51(40):13282-13299

25. Wazzan NA (2015) DFT calculations of thiosemicarbazide, arylisothiocynates, and 1-aryl-2,5-dithiohydrazodicarbonamides as corrosion inhibitors of copper in an aqueous chloride solution. $J$ Ind Eng Chem 26:291-308

26. Akalezi CO, Enenebaku CK, Oguzie EE (2012) Application of aqueous extracts of coffee senna for control of mild steel corrosion in acidic environments. Int J Ind Chem 3(1):13

27. Shi W et al (2013) Molecular dynamics study of polyether polyamino methylene phosphonates as an inhibitor of anhydrite crystal. Desalination 322:137-143

28. Zeng J, Zhang J, Gong X (2011) Molecular dynamics simulation of interaction between benzotriazoles and cuprous oxide crystal. Comput Theor Chem 963(1):110-114

29. Zhao H et al (2014) Quantitative structure-activity relationship model for amino acids as corrosion inhibitors based on the support vector machine and molecular design. Corros Sci $83: 261-271$

Publisher's Note Springer Nature remains neutral with regard to jurisdictional claims in published maps and institutional affiliations. 